Published. Ocean Engineering 42 (2012) 195-204.

\title{
Joint modelling of vertical profiles of large ocean currents
}

\author{
Philip Jonathan \\ Shell Technology Centre Thornton \\ P.O. Box 1 \\ Chester \\ United Kingdom \\ philip.jonathan@shell.com
}

\author{
Kevin Ewans \\ Sarawak Shell Bhd \\ eTiQa Twins (Level 23, Tower 1) \\ 50450 Kuala Lumpur \\ Malaysia \\ kevin.ewans@shell.com
}

\author{
Jan Flynn \\ Shell International Exploration and Production \\ P.O. Box 60 \\ 2280 AB Rijswijk \\ The Netherlands \\ jan.flynn@shell.com
}

September 11, 2011

\begin{abstract}
We present an empirical approach to modelling the vertical (vector) profile of large ocean currents, based on a conditional extremes model (Heffernan and Tawn 2004) from the statistics literature. Observed vector currents at each of a number of water depths are expressed as components with respect to majorand minor-axis of current variation at that depth using Principal Components Analysis. Each current component is then independently decomposed into the sum of (deterministic periodic) tidal and (random) non-tidal currents using a local harmonic model. The marginal and dependence structure of extremes of hourly maxima and minima of non-tidal components is characterised using the conditional extremes model. We simulate under this model to estimate characteristics of extreme current profiles corresponding to arbitrary return periods, and quantify the uncertainty of those estimates. For a sample collected over a 2.5- year period in $250 \mathrm{~m}$ water on the outer shelf of North Western Australia, the model predicts monthly instantaneous extreme conditional profiles well. We also estimate marginal and conditional current profiles corresponding to a 10-year return period.
\end{abstract}

\section{Introduction}

There is scientific and engineering interest in understanding the structure of ocean current profiles, vertically through the water column. Carollo et al. 2005a b provide references to analysis of vertical current structure in the oceanographic literature. From an engineering perspective, current-induced structural loading is an important consideration in offshore and coastal design. Understanding dependence between extreme values of current at different positions in the water column is critical to reliable estimation of extreme loads on oil and gas installations in the deep ocean. Planned measurement programmes were rare until recent years (Griffiths 1996). With more recent widespread adoption of ADCP devices, complete water column measurements of current profile sometimes over several years are available. Reliably estimating extreme current events corresponding to return periods required for engineering design remains a major challenge.

Currents are vector quantities which can be represented as the sum of tidal and non-tidal components. Tidal forcing is essentially deterministic in nature, generated by the gravitational interaction of the Sun, Moon and Earth. Hence tidal currents are periodic with a net velocity of zero over the tidal cycle. Non-tidal currents include permanent currents in the ocean's general circulatory systems and surge currents arising from local meteorological variability of wind and pressure fields. Circulatory currents can be viewed as 
slowly varying deterministic components. Surge currents however are essentially stochastic in nature but show strong seasonal variation. Local bathymetry and vertical stratification of temperature and salinity also influence characteristics of vertical current profiles. Statistical modelling of extreme ocean currents typically proceeds by first removing slowly-varying and periodic components, before applying extreme value methods to residual (surge) current (e.g. Robinson and Tawn 1997). In this way, the extreme value characteristics of (scalar) current components (or speed) can be estimated. Design values corresponding to arbitrary return periods are estimated by simulation, assuming an additive model for total current. By treating current direction as a covariate, a joint model for current speed and direction can also be developed (Robinson and Tawn 1997).

Regarding statistical analysis of extreme vertical current profiles, underlying physics suggests that occurrences of a large current (speed or component) at one depth will be associated with particular current characteristics at other depths. Carollo et al. 2005b report dependence between extremes for close vertical layers, attributed to vertical propagation of flow. Modelling dependence structure of extreme vertical current profiles is essential, therefore, in general, whereas performing independent extreme value analyses per depth would not be appropriate. Extending the Robinson and Tawn 1997 model for current speed and direction for multiple current depths is regrettably not straight forward.

Principal components analysis (PCA, see e.g. Joliffe 2002 sometimes referred to as empirical orthogonal function (EOF) analysis, see e.g. Hannachi et al. [2007]) is commonly used for exploratory data analysis in oceanography (e.g. Liu and Weisberg 2007). Some authors propose using this approach to preprocess residual current prior to statistical analysis. Briefly, a sample of values of two orthogonal residual current components at each of $p$ depths (yielding $2 p$ variables) is re-expressed in terms of projections (or 'scores') onto the set of $2 p$ orthonormal principal component vectors. Each principal component is a linear combination of the original $2 p$ variables. The first principal component is the best one-dimensional summary of the original sample in terms of variance explained (i.e. it is the major axis of the data cloud). Subsequent principal component vectors are defined analogously, such that the first and second principal components provide the best two-dimensional summary, etc. Extreme value analysis can then be performed on the more informative principal component scores variables. Strictly, all principal component scores variables should be analysed to avoid loss of information. Return values estimated again assuming additivity of scores variables. Critically however, scores variables (established using the full sample) are not generally independent in the extremes. Hence, joint modelling of scores variables would still be necessary to characterise extremal behaviour adequately.

Direct joint modelling of extremes of two or three variables is sometimes possible, with limitations (see Jonathan et al. 2010). Heffernan and Tawn 2004 present a conditional extreme value model. Using asymptotic arguments, they derive a parametric equation for the form of one or more variables conditional on a large value of another, valid for extremes from a wide class of multivariate distributions, providing a practical basis for joint modelling of extremes in high dimensions.

Any reasonable approach to modelling vertical residual current profiles must accommodate (a) the vector nature of currents at each depth, and (b) the dependence between currents at different depths. In this work, we choose to model maxima and minima for current components in orthogonal directions over short intervals of time, rather than instantaneous orthogonal current components (or speed and direction). That is, we summarise the variation in orthogonal current components $c_{a}(t), c_{b}(t), t \in$ interval $T$, using the quartet $c_{a k}^{+}, c_{a k}^{-}, c_{b k}^{+}, c_{b k}^{-}$, where ${ }^{\prime}+{ }^{\prime}$ represents the maximum observed during interval $T$, and ' $-^{\prime}$ the minimum, for the corresponding variable. This summary representation provides a pragmatic approach to joint modelling of extremes of a set of two-dimensional vectors, which we demonstrate does not compromise the quality of inference unduly for appropriate choice of interval length.

The layout of the paper is as follows. In section 2, we introduce the motivating example for the current work and illustrate the pre-processing steps undertaken. We outline the local harmonic model used to remove slowly-varying and periodic current components, and the effect of sampling hourly maxima and minima. In section 3, the conditional extremes model is outlined, and results of its application to residual currents reported. In section 4 , we report the results of a simulation study to generate extreme conditional profiles, and compare simulation with measurement. Section 5 provides a brief discussion and conclusions. The purpose of this paper is to demonstrate the application of the conditional model for estimation of extreme vertical profiles of residual current. Emphasis is therefore placed on extreme value analysis. For completeness, however, we also illustrate how results from different modelling stage are combined to provide 
estimates of extreme vertical profiles of total current.

\section{$2 \quad$ Data and pre-processing}

\subsection{Location, measurement and current regime}

Measurements were made in approximately $250 \mathrm{~m}$ water depth in the Browse Basin on the northwest continental shelf of Western Australia. The location of interest lies in the shelf break region, close the continental slope, with steeply sloping bathymetry. The slope is oriented approximately NE-SW in the vicinity of the measurements. The current meter mooring comprised 8 CM04 single-point acoustic current meters, positioned at $40 \mathrm{~m}$ intervals through the water column, with the exception of the near-bed current meters, which were positioned at $3 \mathrm{~m}$ and $1 . \mathrm{m}$ above seabed. The instruments sampled current velocity and sea water temperature at $2 \mathrm{~Hz}$, with a vector-average current velocity calculated at 1-minute intervals. The mooring was deployed for a period of just over $21 / 2$ years, with service visits at approximately 6 -monthly intervals. We consider resulting 1-minute measurements of Easterly and Northerly current components at depths $D_{1}-D_{8}$, respectively $27 \mathrm{~m}, 67 \mathrm{~m}, 107 \mathrm{~m}, 147 \mathrm{~m}, 187 \mathrm{~m}, 227 \mathrm{~m}, 254 \mathrm{~m}$ and $255 \mathrm{~m}$ below the surface.

In the Browse Basin, tides are predominantly semidiurnal. Accordingly, barotropic tide typically exhibits four current reversals (and four current speed peaks) each day. There is a strong semidiurnal tidal signal in the current direction. Currents rotate anti-clockwise from west, south, east through north in the upper water column. At the seabed, current directions are constrained to the ebb and flood directions, which are roughly northwest and southeast, perpendicular to the axis of the continental slope. Browse Basin waters are also typically strongly temperature-stratified in summer, so that baroclinic tide is important. The importance of baroclinic tide is enhanced by steeply sloping bathymetry, and the positioning of the measurement location in the shelf break region.

Local wind-forcing is usually most pronounced under severe tropical cyclones. After cyclone passage, inertial currents with inertial period of approximately 50 hours may be initiated. Since the wind regime is distinctly seasonal, near-surface currents may display similar seasonality, with summer easterly to northeasterly currents, and winter westerly to south-westerly currents. Local wind-driven surface currents may attain maximum speeds of $0.7 \mathrm{~ms}^{-1}$ during extreme monsoonal or Trade Wind surges. More typical speeds would be in the range $[0.2,0.4] \mathrm{ms}^{-1}$. The direction of flow would be slightly to the left of the line of forcing due to the Coriolis effect, but generally along the bathymetric contours. During severe tropical cyclones, forcing would be much stronger, with local current direction depending upon the relative location of the centre of the storm. Tropical cyclone generated current speeds will typically exceed $1.0 \mathrm{~ms}^{-1}$ and can approach or even exceed $2.0 \mathrm{~ms}^{-1}$, for the near-surface layer.

Regional circulation involves the general anticlockwise gyre of the Indian Ocean, seasonal influences such as prevailing mean wind, other regional influences such as the Pacific-Indian Ocean through-flow, and any contribution from continental shelf waves. The Indian Ocean anticlockwise gyre involves a westward flowing South Equatorial Current with speeds attaining $0.4 \mathrm{~ms}^{-1}$ at the location. Source water for the Pacific-Indian Ocean through-flow is relatively warm, low salinity water amassed against the eastern boundaries of the Indonesian Archipelago by the Trade Winds of the Pacific Ocean. This water filters through the archipelago, feeding the South Equatorial Current and flooding the continental shelf of Western Australia. Since the volume of available source water is strongly correlated with El Nio Southern Oscillation (ENSO) events, the currents at the measurement location are expected to show some inter-annual variability on the same timescale as for ENSO events.

Due to the proximity of the measurement location to the shelf slope and steep bathymetry, high frequency currents associated with the passage of solitons are anticipated. These are characterised by speed spikes in the range $[0.5,0.7] \mathrm{ms}^{-1}$, particularly near the seabed, and temperature spikes at the thermocline. Soliton events occur more frequently in and around April, when thermal stratification is most intense.

\subsection{Components along major- and minor-axis of current ellipse}

At each depth $D_{k}, k=1,2, \ldots, 8$, consider resolved values of current along the major and minor axes of the total current ellipse, $c_{M k}$ and $c_{m k}$ respectively. Figure 1 shows the direction of the major axis as a function of depth. The major axis is approximately perpendicular to the shore line. We also observe an anti-clockwise 


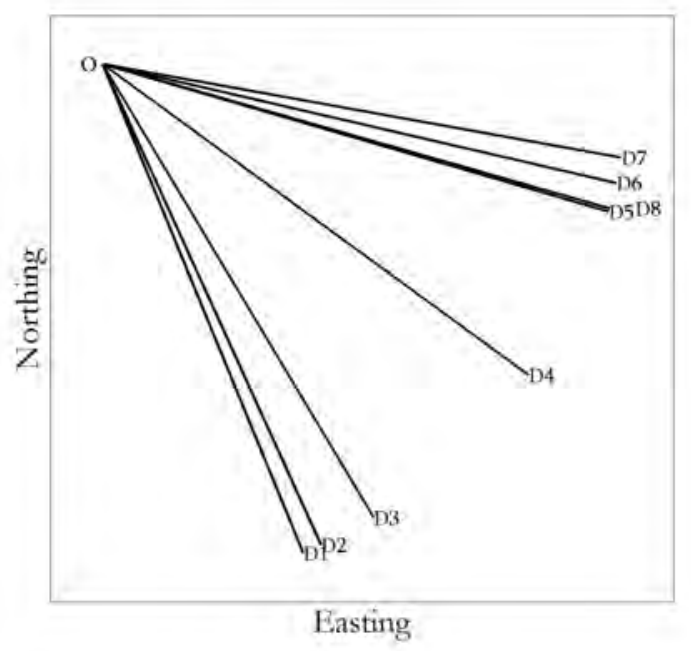

Figure 1: Direction of major axis of current ellipse for each of 8 depths $D_{1}-D_{8}$. The major axis is approximately perpendicular to the slope line. Note anti-clockwise rotation of major axis with increasing depth for $D_{1}-D_{5}$.

rotation of the major axis of total current with increasing depth from $27 \mathrm{~m}$ to $187 \mathrm{~m}$. It is tempting to conclude that this might indicate an Ekman drift effect, and that mean current flow has a strong wind drift component. However, Larsen et al. 2000 report a clockwise rotation of the major axis of the tidal current ellipse with depth for observations in the Iceland-Scotland ridge. As the currents at our location are similarly tidally dominated, we are in fact observing, perhaps topographic, steering of tidal components with depth. Figure 2 shows the variation of $c_{M 4}$ in time for the first 100 days of observation; tidal effects are obvious in the major-axis components $c_{M}$ • at each depth, but less clear for the minor axis components $c_{m}$ (shown in Figure 3 for $D_{4}$ ). Note that henceforth we use components $c_{M 4}$ (and $c_{m 4}$ ) as 'typical' examples to illustrate discussion as necessary.

Figure 4 shows that current variance reduces as a function of increasing water depth, for total current, $c$, and current components $c_{M}, c_{m}$. Component $c_{M}$ explains approximately $3 / 4$ of current variance at each water depth.

Note that, in this work, principal components analysis is used independently at each depth to identify resolved current components corresponding to the major and minor axes of current variation.

\subsection{Tidal and residual currents}

We decompose each of $c_{j k}, j=M, m, k=1,2, \ldots 8$, into tidal (subscript ' $T$ ') and residual (subscript ' $R^{\prime}$ ) components $\left(c_{T j k}, c_{R j k}\right)$ using a local harmonic model. After some exploration, we found that an additive model of the form:

$$
c_{j k}(t)=a(t)+b_{1} \sin \left(\left(24 / T_{1}\right) t+\phi_{1}\right)+b_{2} \sin \left(\left(24 / T_{2}\right) t+\phi_{2}\right)
$$

was sufficient to explain tidal effects over a moving 2-day window, with $T_{1}=12.42$ hours (principal lunar constituent, $M_{2}$ ) and $T_{2}=12$ hours (principal solar constituent, $S_{2}$ ). Figure 5 illustrates the result of decomposition of component $c_{M 3}$. Major tidal variation has been captured in the tidal component. We note that slowly-varying current is captured by the local intercept term, $a(t)$, important in the case of $c_{m 3}$ (see Figure 6).

Note that, in this work, a simple local harmonic model is found to explain deterministic variation in current components well. In general, other model forms (see, e.g., Robinson and Tawn 1997, Pawlowicz et al. 2002) might be more appropriate. 


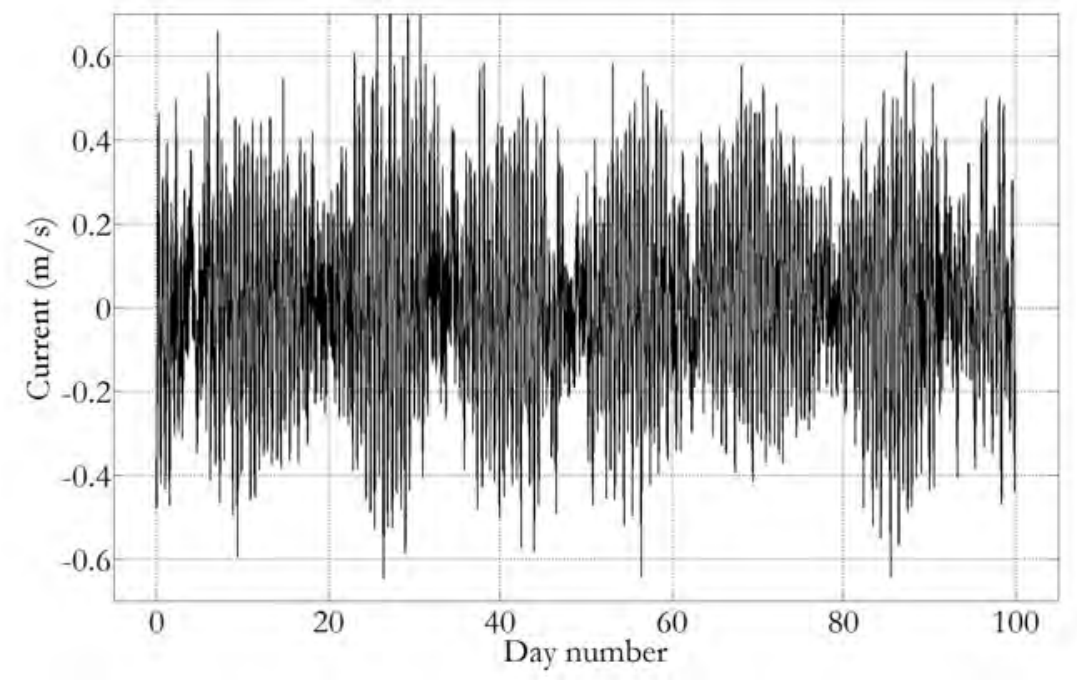

Figure 2: Time-series of major-axis component at depth $D_{4}, c_{M 4}$ for first 100 days of observation. Periodic tidal effects obvious.

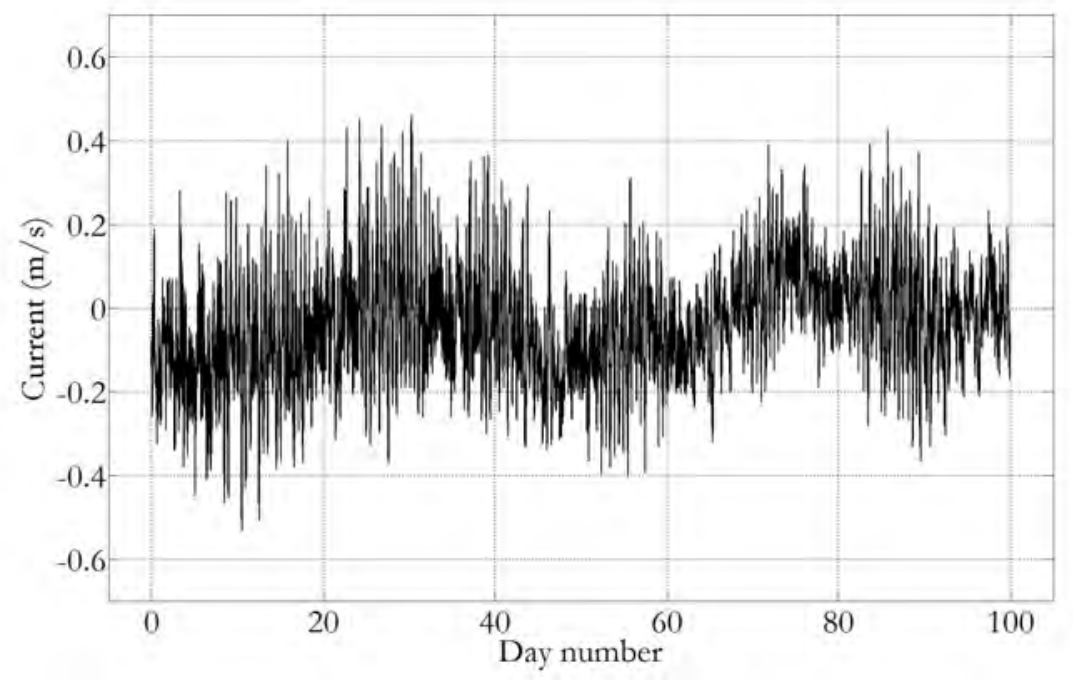

Figure 3: Time-series of minor-axis component at depth $D_{4}, c_{m 4}$ for first 100 days of observation. Periodic tidal effects less prominent than for $c_{M 4}$. 


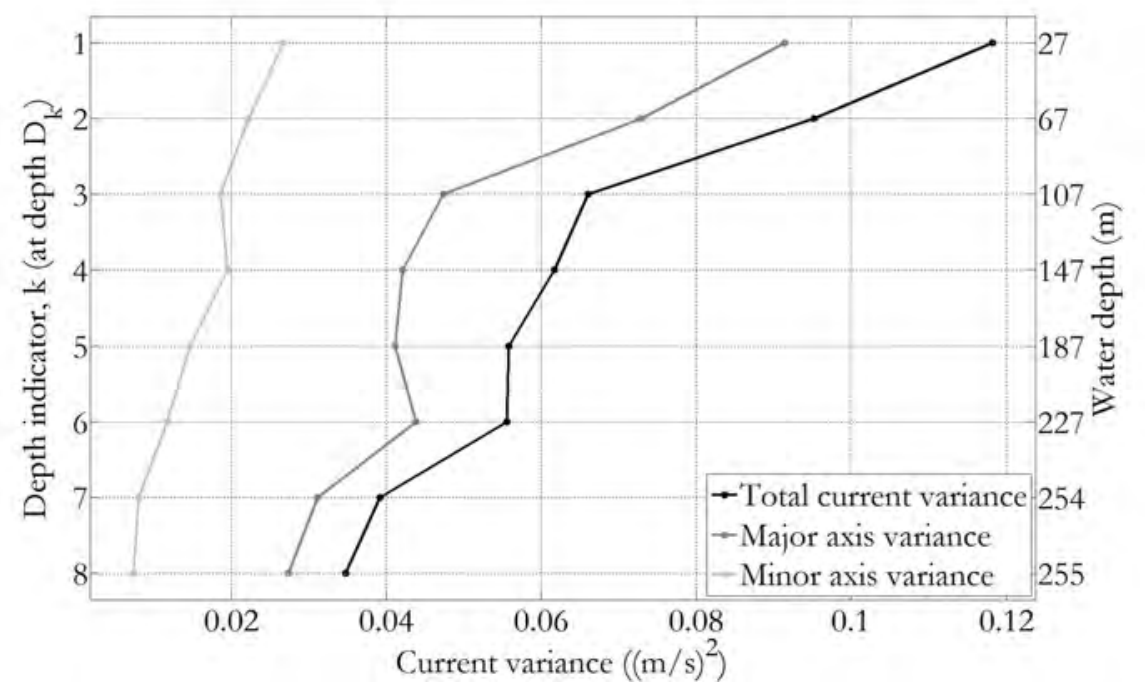

Figure 4: Current variance with water depth, for total current $c$ (solid), major-axis component $c_{M} \bullet$ (dark grey) and minor-axis component $c_{m}$ ( (light grey). Current variance reduces with increasing depth, with $c_{M} \bullet$ explaining approximately $3 / 4$ of current variance at each depth.

(a) Tidal

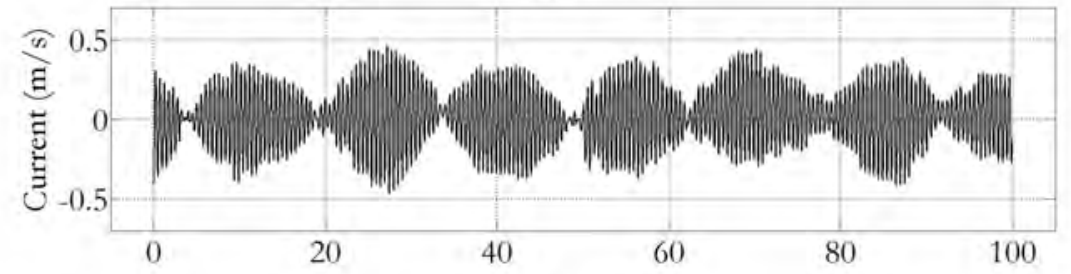

(b) Residual

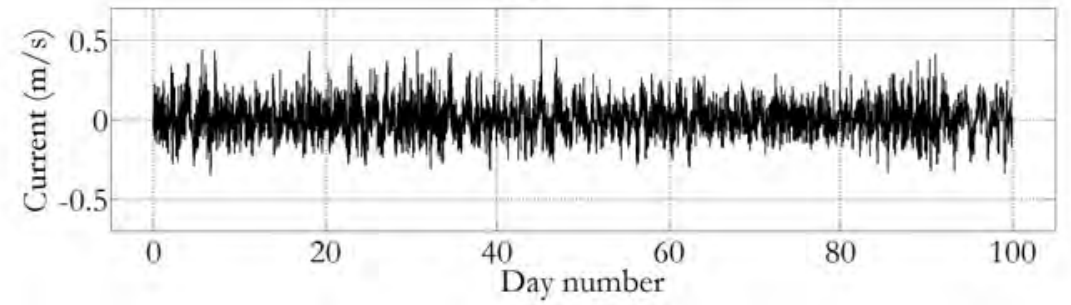

Figure 5: Decomposition of major-axis current at depth $D_{4}, c_{M 4}$, into tidal (including slowly-varying) and residual currents for first 100 days of observation. Major tidal variation is captured by local model. 
(a) Tidal

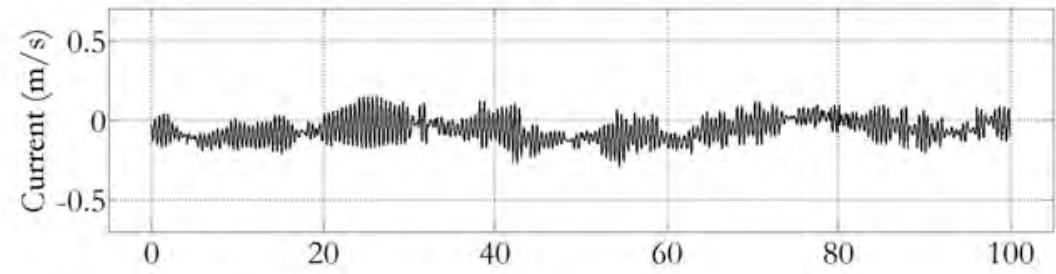

(b) Residual

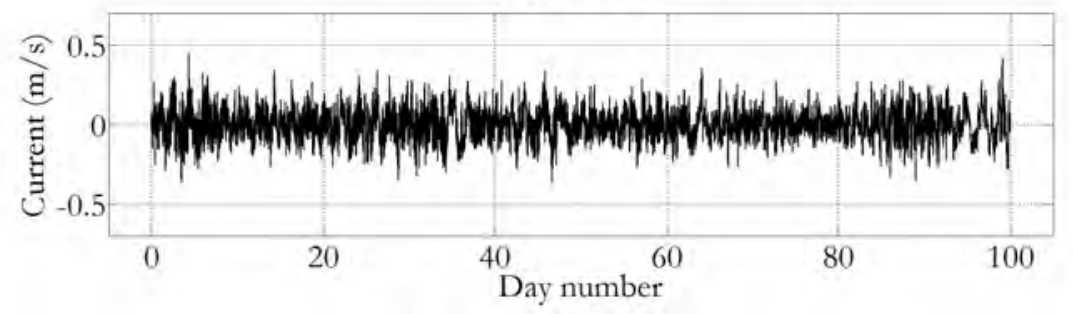

Figure 6: Decomposition of minor-axis current at depth $D_{4}, c_{m 4}$, into tidal (including slowly-varying) and residual currents for first 100 days of observation. Local model captures slowly-varying term.

\subsection{Hourly extreme currents}

Hourly maxima $c_{i j k}^{+}$and minima $c_{i j k}^{-}$for each component $c_{i j k}, i=\{T, R\}, j=\{M, m\}, k=1,2, \ldots, 8$ were isolated. Hourly extremes of residual current are used for extreme value analysis. Hourly extreme of tidal current are necessary for recombination with extreme hourly residual currents during simulation to estimate return values in section 4 . Figure 7 shows time-series of hourly maxima and (negative) minima for $c_{T M 3}$ and $c_{R M 3}$. Note that "hourly minima" are defined using $c_{\bullet}^{-}=\max \left\{-c_{\bullet}\right\}$ over the hour interval, so that we are always interested in large positive values of extremes. Since the major-axis of the current ellipse is approximately perpendicular to the slope, we can therefore think of $c_{\bullet}^{+}$as the 'hourly maximum onshore' component and $c_{\bullet}^{-}$as the 'hourly maximum offshore' component. An interval of one hour provides sufficient current observations to estimate component maxima and minima reasonably, whilst allowing characterisation of the variation of these quantities in time.

Figure 8 shows a Spearman rank correlation map of the hourly maximum / minimum residual currents. The Spearman rank correlation between values for two variables in a sample is the correlation between the sorted values of those variables; rank correlation is less sensitive to individual 'outlier' points than it's conventional (Pearson) equivalent. Positive rank correlations are shown in the North-West section of the map, negative rank correlations in the South-East. Hourly maxima $c_{R M k}^{+}$are positively inter-correlated for $k \geq 5$, as are $c_{R M k}^{-}, c_{R m k}^{+}$and $c_{R m k}^{-}$. These inter-correlations are indicative of dependence structure which needs to be modelled adequately, especially if also evident in extremes of the variables. Hourly maxima $c_{R j k}^{+}$and minima $c_{R j k}^{-}$are also positively correlated for given component $j(=\{M, m\})$ and $k(=1,2, \ldots, 8)$, suggesting that hours with large maxima residuals also have large values of (negative) minima. Other inter-correlations appear to be relatively small.

Some or all non-negligible inter-correlations in Figure 8 could point to local non-tidal influences, including effects of wind- and pressure-fields, temperature, salinity and bathymetry. They could also indicate inadequate harmonic analysis. The usual approach (Pugh 1987) is to perform a full harmonic analysis on $c_{M}$ and $c_{m}$ components per depth independently, perhaps using of a pre-filter (Graff 1986) to eliminate long-period variations. Here, we have used a local harmonic model to capture major local harmonic effects, also fitted independently per variable. It is apparent that lack of fit of a harmonic model of data from dependent variables (especially if applied independently per variables) will give rise to inter-correlated residual currents. We suspect therefore that correlation maps similar to that shown in Figure 8 would arise as the result of a typical harmonic analysis of dependent ocean current data. Regardless, it is important that the modelling approaches adopted accommodate dependence structure adequately. 
(a) Maximum tidal

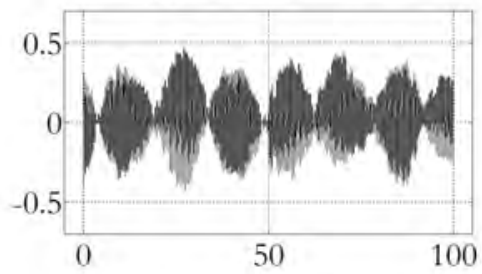

(c) Maximum residual

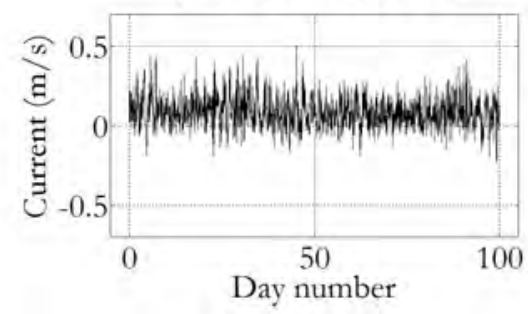

(b) (Negative) minimum tidal

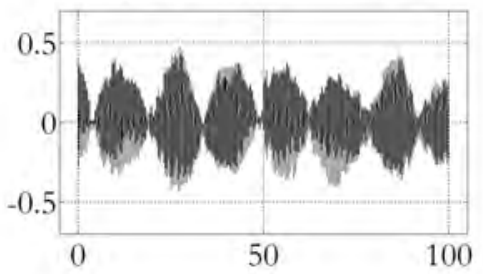

(d) (Negative) minimum residual

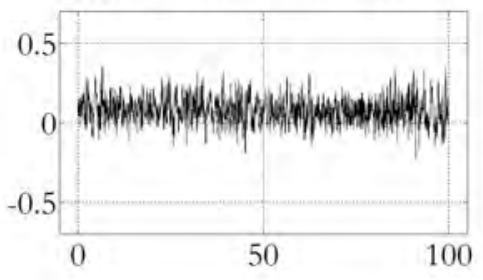

Figure 7: Time-series of hourly maxima and (negative) minima for major-axis currents at depth $D_{4}$, corresponding to tidal (including slowly-varying) and residual components. From top to bottom: $c_{T M 4}^{+}, c_{T M 4}^{-}$, $c_{R M 4}^{+}$and $c_{R M 4}^{-}$

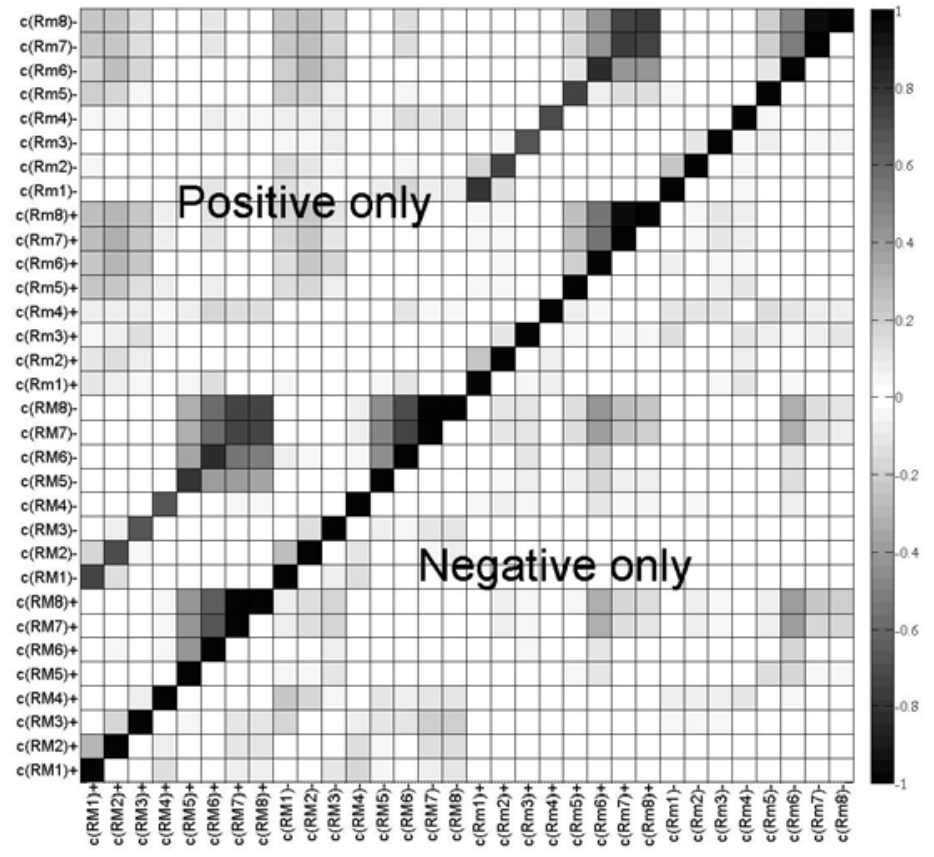

Figure 8: Spearman rank correlation map for hourly maxima and (negative) minima of residual current components. Positive rank correlations are shown in the North-West, negative rank correlations in the South-East. Remaining correlations are predominantly positive by construction. 
We are interested in quantifying extreme current profiles in the water column. Assuming tidal components to be effectively deterministic, we concentrate on characterising the joint extremes of hourly extreme residual currents. We assume, for arbitrarily long return periods, that realisations of tidal components can be added to realisations of residual components (from appropriate extreme value models) to estimate extremes of the sum of tidal and residual components. The conditional extremes model introduced in section 3 provides a natural framework for joint modelling of the full set $c_{R j k}^{+/-}, j=\{M, m\}, k=1,2, \ldots, 8$ of 32 hourly extreme residual currents.

\section{Conditional extremes model}

This section describes the conditional extremes model for jointly modelling extreme residual currents. For a vector $\mathbf{Y}\left(=\left\{Y_{j}\right\}_{j=1}^{p}\right)$ of random variables with marginal Gumbel distributions, Heffernan and Tawn 2004 derive a parametric form for the conditional distribution of the remaining variables $\mathbf{Y}_{-k}\left(=\left\{Y_{j}\right\}_{j=1, j \neq k}^{p}\right)$ given a large value $y_{k}$ of the variable $Y_{k}$, at least as large as threshold $v_{k}, k=1,2, \ldots, p$. This parametric form, motivated by the assumption of a particular limit representation for the conditional distribution (see e.g., discussion section of Jonathan et al. 2010]) is appropriate to characterise the conditional behaviour of a wide range of theoretical examples of multivariate distributions for extremes. Model form for positively dependent variables $\mathbf{Y}$ takes a particularly simple form:

$$
\left(\mathbf{Y}_{-k} \mid Y_{k}=y_{k}\right)=\mathbf{a}_{k} y_{k}+y_{k}^{\mathbf{b}_{k}} \mathbf{Z}_{k}, \text { where } y_{k} \geq v_{k}, \text { for } k=1,2, \ldots, p
$$

where $\mathbf{a}_{k}\left(=\left\{a_{j k}\right\}_{j=1, j \neq k}^{p}\right)$ and $\mathbf{b}_{k}\left(=\left\{b_{j k}\right\}_{j=1, j \neq k}^{p}\right)$ are vectors of location and scale parameters respectively to be estimated, with element $a_{j k} \in[0,1]$ and $b_{j k} \in(-\infty, 1)$, and $y_{k}$ large, for $j=1,2, \ldots, p, j \neq k$, and $k=1,2, \ldots, p$, and component-wise multiplication is understood (i.e. for $p$-dimensional vectors $\mathbf{r}=\left\{r_{i}\right\}_{i=1}^{p}$ and $\mathbf{s}=\left\{s_{i}\right\}_{i=1}^{p}$, then $\left.\mathbf{r s}=\left\{r_{i} s_{i}\right\}_{i=1}^{p}\right) . \mathbf{Z}_{k}$ is a vector random variable, independent of $Y_{k}$, converging with increasing $y_{k}$ to a non-degenerate limiting distribution $G_{k}$. For each $k, k=1,2, \ldots, p$, joint tail behaviour conditional on $Y_{k}$ is characterised by $\mathbf{a}_{k}, \mathbf{b}_{k}$ and $G_{k}$. The form of distribution $G_{k}$ is not specified by theory. When two variables $Y_{j}, Y_{k}$ are negatively dependent (i.e. $a_{j k}=0, b_{j k}<0$ ), the form of the conditional relationship is slightly different, but still amenable to empirical modelling:

$$
\left(\mathbf{Y}_{-k} \mid Y_{k}=y_{k}\right)=\mathbf{c}_{k}-\mathbf{d}_{k} \log _{e}\left(y_{k}\right)+y_{k}^{\mathbf{b}_{k}} \mathbf{Z}_{k}, \text { where } y_{k} \geq v_{k}, \text { for } k=1,2, \ldots, p
$$

where $\mathbf{c}_{k}\left(=\left\{c_{j k}\right\}_{j=1, j \neq k}^{p}\right)$ and $\mathbf{d}_{k}\left(=\left\{d_{j k}\right\}_{j=1, j \neq k}^{p}\right)$ have elements such that $c_{j k} \in(-\infty, \infty)$ and $d_{j k} \in$ $[0,1]$. In the current work, the vast majority of pairwise dependencies between variables is positive. For a sample $\left\{y_{i j}\right\}_{i=1, j=1}^{n, p}$ of values from $\mathbf{Y}$ with values of conditioning variate $Y_{k}$ exceeding an appropriate threshold $v_{k}$, the values of $\mathbf{a}_{k}, \mathbf{b}_{k}$ and $G_{k}$ are estimated using regression. For simplicity and computational ease during model fitting, $G_{k}$ is assumed to be a multivariate normal distribution with mean $\mu_{\mathbf{Z} k}$ and diagonal variance-covariance matrix with elements $\sigma_{\mathbf{Z} k}^{2}$ treated as nuisance parameters, for $k=1,2, \ldots, p$. These assumptions resolve the modelling procedure into fitting of $p-1$ pairwise models for pairs $\left(Y_{j}, Y_{k}\right)$, $j=1,2, \ldots, p, j \neq k$, for each of $p$ conditioning variates $Y_{k}, k=1,2, \ldots, p$. Fitted residuals:

$$
\hat{z}_{i j k}=\frac{\left(y_{i j}-\hat{a}_{j k} y_{i k}\right)}{y_{i k}^{\hat{b}_{j k}}}, i=1,2,3, \ldots, n, j=1,2, \ldots, p, j \neq k, k=1,2, \ldots, p
$$

provide a dependent sample from the multivariate distribution $G_{k}$, which is then resampled (with replacement) in subsequent simulations. The adequacy of model fit can be assessed by (1) demonstrating that the values $\left\{\hat{z}_{i j k}\right\}_{i=1}^{n}$ and $\left\{y_{i k}\right\}_{i=1}^{n}$ are not obviously dependent (thus violating a modelling assumption), (2) exploring the effect of varying $v_{k}$ on $\hat{a}_{j k}, \hat{b}_{j k}$ and subsequent estimates (e.g. of probabilities associated with extreme sets), and (3) bootstrap resampling to estimate the uncertainty of estimates for $\mathbf{a}_{k}, \mathbf{b}_{k}$ and subsequent estimates for a given threshold choice $v_{k}$ (see e.g. Jonathan et al. [2010]).

\subsection{Marginal modelling}

The generalised Pareto (GP) form is appropriate for modelling marginal distributions of peaks over threshold, rather than the Gumbel distribution. Therefore to use the conditional model above we need to first model 
the original variables $\mathbf{X}\left(=\left\{X_{j}\right\}_{j=1}^{p}\right)$ marginally using GP, then transform from GP to Gumbel using the probability integral transform as follows. Suppose we fit the GP distribution (to the sample from $X_{k}$ without loss of generality, $k=1,2, \ldots, p)$ :

$$
F_{G P}\left(x ; \xi_{k}, \beta_{k}, u_{k}\right)=1-\left(1+\frac{\xi_{k}}{\beta_{k}}\left(x-u_{k}\right)\right)_{+}^{-\frac{1}{\xi_{k}}}
$$

and estimate cumulative probabilities $\left\{F_{G P}\left(x_{i k} ; \hat{\xi}_{k}, \hat{\beta}_{k}, u_{k}\right)\right\}_{i=1}^{n}$. Now the standard Gumbel distribution has cumulative distribution function:

$$
F_{G}(x)=\exp (-\exp (-x))
$$

Thus if we define the transformed sample $\left\{y_{i k}\right\}_{i=1}^{n}$ such that $F_{G}\left(y_{i k}\right)=F_{G P}\left(x_{i k} ; \hat{\xi}_{k}, \hat{\beta}_{k}, u_{k}\right)$ or:

$$
y_{i k}=-\log \left(-\log \left(F_{G P}\left(x_{i k} ; \hat{\xi}_{k}, \hat{\beta}_{k}, u_{k}\right)\right)\right) \text { for } i=1,2,3, \ldots, n
$$

the transformed sample will correspond to a Gumbel distribution. Similarly, given any value $y$ (of Gumbel variate), we can calculate the corresponding value $x$ on the original GP scale. It is essential to demonstrate the adequacy of GP marginal fits, e.g. by using the mean residual life plot and stability of marginal shape parameter and suitable extreme quantiles as a function of threshold.

\subsection{Conditional simulation}

To simulate a random drawing $\left\{x_{s j}\right\}_{j=1}^{p}$ from the conditional distribution $\mathbf{X}_{-k} \mid X_{k}>u_{k}$, the following procedure can then be followed:

1. draw a value $y_{s k}$ of $Y_{k}$ at random from the standard Gumbel distribution,

2. draw a set of values $\left\{z_{s j k}\right\}_{j=1, j \neq k}^{p}$ of $\mathbf{Z}_{-k}$ at random from the set $\left\{\hat{z}_{i j k}\right\}_{i=1, j=1, j \neq k}^{n, p}$ generated during model fitting,

3. calculate that value of $y_{s j} \mid y_{s k}=\hat{a}_{j k} y_{s k}+y_{s k}^{\hat{b}_{j k}} z_{s j k}$ for $j=1,2, \ldots, p, j \neq k$,

4. transform $\left\{y_{s j}\right\}_{j=1}^{p}$ to $\left\{x_{s j}\right\}_{j=1}^{p}$ using the probability integral transform and the estimated GP marginal model parameters.

To use this scheme to generate a multivariate dependent sample, we must first estimate the relative frequency with which to condition on each variable $Y_{k}$ (or $\left.X_{k}\right), k=1,2, \ldots, p$, in turn. This is achieved as follows. Note that the sample $\left\{y_{i j}\right\}_{i=1, j=1}^{n, p}$ corresponds to a subset $E$ of the original sample, for which the value of at least one variable (for each observation) exceeds its corresponding threshold ( $v_{k}$ for variable $k$ ). We can therefore partition $E$ into the union of sets $\left\{E_{k}\right\}_{k=1}^{p}$ :

$$
E_{k}=\left\{\left\{y_{i j}\right\}_{i=1, j=1}^{n, p} \text { s.t. } y_{i k}>y_{i j} ; j=1,2, \ldots, p, j \neq k ; i=1,2, \ldots, n\right\}
$$

so that for observations in set $E_{k}$, the value of variable $k$ is more extreme in its marginal distribution than the value of any other variable. Since the conditional extremes model is motivated asymptotically, it is most appropriately applied to the conditioning variate whose value is most extreme in its distribution. We therefore use the relative frequency of observations in the sets $\left\{E_{k}\right\}_{k=1}^{p}$ to determine the rate at which we condition on each variable $Y_{k}, k=1,2, \ldots, p$, during conditional simulation. See Heffernan and Tawn 2004] for further discussion.

This simulation procedure is used to generate multivariate time-series of the 32 hourly extreme residual currents $c_{R j k}^{+/-}, j=\{M, m\}, k=1,2, \ldots, 8$ with the appropriate marginal extreme and conditional extreme dependence structures. 


\section{Application}

\subsection{Modelling approach}

The approach to modelling extremes of vector current with depth can be summarised as follows:

1. Time series of vector currents at each depth are resolved into uncorrelated major- and minor-axis components (as illustrated in section 2.2) using principal components analysis.

2. Each major- and minor-axis component is decomposed into 'deterministic' tidal (including slowly varying) and 'random' residual time-series, as explained in section 2.3

3. It is assumed that the tidal components of current (estimated from the original sample using local harmonic analysis) are sufficient to characterise the (joint) distributions of these components (but see discussion in section 5). No further modelling of tidal components is therefore necessary; the distribution of (hourly extreme) tidal components corresponding to arbitrarily long return periods can be estimated by resampling with replacement from the estimated tidal component data.

4. Hourly extreme residual series from major- and minor-axis components are estimated empirically (as discussed in section 2.4).

5. Series for hourly extreme residuals are observed to be approximately stationary and homogeneous in time. Some series exhibit positive interdependence (as seen in Figure 8). This suggests that we model the full set of hourly extreme residuals (for major- and minor-axis components at all water depths) using the conditional extremes model introduced in section 3. (A straight forward application of the conditional extremes model to oceanographic data is given in Jonathan et al. 2010]). We can then simulate under the estimated model to characterise the distribution of hourly extreme residuals corresponding to arbitrarily long return periods.

6. Realisations of residual and tidal currents are added to estimate hourly extreme currents in terms of maxima and (negative) minima of major- and minor-axis components.

\subsection{Applying the conditional extremes model}

In this section, we apply the conditional extremes model to hourly extreme residual current series for majorand minor-axis components for measurements from the outer shelf of North Western Australia introduced in section 2. We perform conditional simulations using the model to estimate the characteristics of marginal and conditional profiles corresponding to a 10-year return period. We also compare estimates of conditional profiles corresponding to monthly maxima from simulation and observation.

Firstly, threshold exceedences of each of the 32 hourly extreme residual currents $c_{R j k}^{+/-}, j=\{M, m\}, k=$ $1,2, \ldots, 8$ are modelled in turn using the GP distribution introduced in section 3.1. It was found that a threshold corresponding to a non-exceedence probability of 0.95 yielded reasonably stable results in all cases (assessed, e.g., by inspecting the variation of the estimated GP shape parameter $(\xi)$ as a function of threshold for thresholds near that chosen). Estimated values for $\xi$ and GP scale parameter $(\beta)$ are illustrated in Figure 9 as a function of depth. We observe that estimated values of $\xi$ are generally negative, indicating the existence of finite upper limit for the estimated distribution. The high values of $\xi$ for near-bottom currents and the implied longer tail of the distribution are indicative of the occurrence of relatively infrequent but high intensity current events near to the bottom. We speculate that these are associated with near-bottom enhancement of solitons induced by internal tides under certain conditions at spring tide.

Using the estimated GP models, sample data for each of the 32 hourly extreme residual currents were transformed to Gumbel scale using the probability integral transform (see section 3). Then, conditioning on each of the 32 variates in turn, the conditional extremes model was estimated. Again, a threshold corresponding to a non-exceedence probability of 0.95 yielded reasonably stable results in all cases. The variability of estimated values of model coefficient, $a$, and exponent, $b$ in each case to small changes in threshold (near that chosen) was found to be small in most cases. The precision of estimation for given threshold can be estimated using bootstrapping (as illustrated in Jonathan et al. [2010]) if required. In this work, diagnostic plots (as suggested in 3) were inspected to assure general adequacy of fit. Figure 10 
(a)

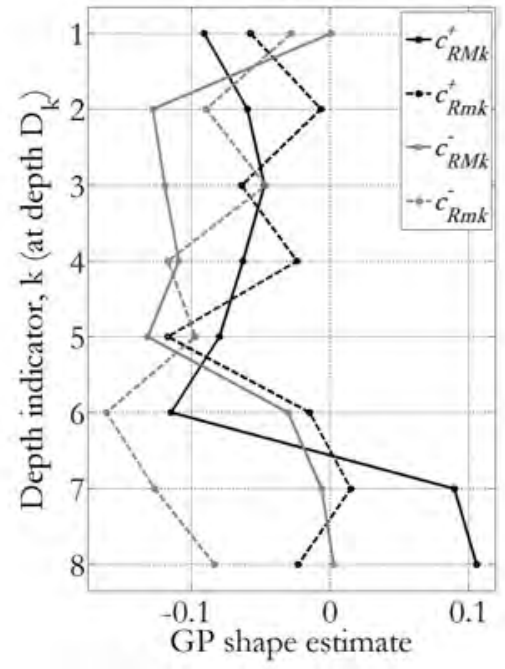

(b)

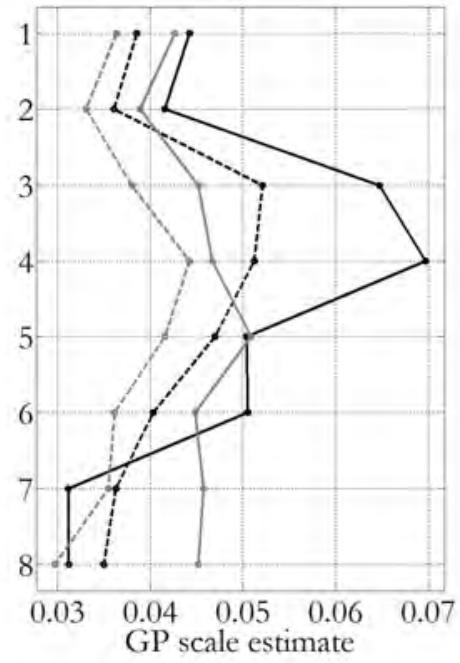

Figure 9: Estimated values for parameters of marginal generalised Pareto models for hourly extreme residual currents with depth $(k=1,2, \ldots, 8)$ : (a) shape parameter, and (b) scale parameter. Lines give estimates for maxima and (negative) minima of major- and minor-axis components. A threshold corresponding to non-exceedence probability of 0.95 was selected throughout.

shows estimates for $a$ and $b$ as a function of water depth, for $c_{R M 4}^{+}$as conditioning variate. Note that the estimates for $a$ are generally positive, indicated that extremes are positively associated, but that most values are $<0.2$ indicating that association is weak. The estimates for $b$ are within a relatively narrow interval (of approximately $(-0.2,0.5))$. Qualitatively similar results were obtained for the other 31 conditioning variates.

\subsection{Estimation of return values}

The estimated marginal and conditional extremes models provide a basis for simulating arbitrarily long time-series of hourly extreme residual currents, with the appropriate marginal and conditional dependence structures. Using the procedure outlined in section 3.2. we simulate 100 years of hourly extreme residual currents $c_{R j k}^{+/-}, j=\{M, m\}, k=1,2, \ldots, 8$. Figure 11 gives marginal 10-year return values for hourly extreme residual currents $c_{R M k}^{+}, c_{R m k}^{+}, c_{R M k}^{-}$and $c_{R m k}^{-}$with depth $(k=1,2, \ldots, 8)$ estimated by simulation, summarised in terms of the median, $25 \%$ ile and $75 \%$ ile values. For comparison (and partial validation of the simulation procedure), corresponding values calculated from theory using fitted marginals are also shown. It is interesting that the values of $c_{R M k}^{+}$are larger at depths 4 and 5 , but these estimates should not be over-interpreted in isolation of tidal (and slowly-varying) components.

Figure 12 shows conditional values of $c_{R M k}^{+}, c_{R m k}^{+}, c_{R M k}^{-}$and $c_{R m k}^{-}$with depth $(k=1,2, \ldots, 8)$ for exceedences of the 10-year return level of $c_{R M 4}^{+}$. It appears that other components are not strongly dependent on the conditioning variate. Results for conditioning on exceedences of the 10-year return level of $c_{R M 1}^{+}$(not shown) indicate stronger dependence between depths $D_{1}, D_{2}$ and $D_{3}$.

A simulation of 100 years of hourly extremes tidal (and slowly-varying) components $c_{T j k}^{+/-}, j=\{M, m\}, k=$ $1,2, \ldots, 8$ was generated using the procedure outlined in section 4.1 , its realisations combined with those from the conditional simulation of residual components to yield a simulation of 100 years of hourly extreme total current components. This simulation is the basis for all estimates of marginal and conditional return values below. Figure 13 gives marginal 10-year return values for hourly extreme total currents $c_{M k}^{+}, c_{m k}^{+}, c_{M k}^{-}$and $c_{m k}^{-}$with depth $(k=1,2, \ldots, 8)$, summarised in terms of the median, $25 \%$ ile and $75 \%$ ile values. We see that the value of each total current component reduces with increasing depth. The 10-year marginal return value of $c_{M k}^{+}$at depths $D_{1}-D_{4}$ is approximately $1.0 \mathrm{~ms}^{-1}$, whereas for depths $D_{5}-D_{8}$ its value is approximately $0.8 \mathrm{~ms}^{-1}$.

Figure 14 shows conditional values of $c_{M k}^{+}, c_{m k}^{+}, c_{M k}^{-}$and $c_{m k}^{-}$with depth $(k=1,2, \ldots, 8)$ for exceedences 
(a)

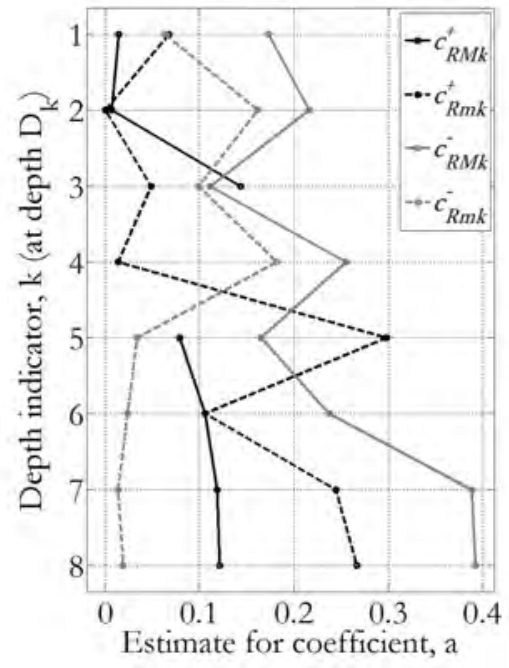

(b)

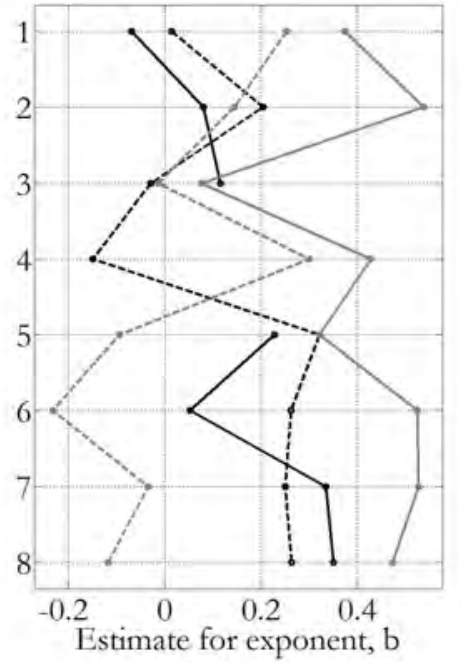

Figure 10: Estimates for conditional extremes model parameters $a$ and $b$ (see Equation 2 for hourly extreme residual series with $c_{R M 4}^{+}$as conditioning variate with depth $(k=1,2, \ldots, 8)$. Positive dependence $(a>0)$ is typically observed for all conditioning variates. A threshold corresponding to non-exceedence probability of 0.95 was selected throughout.

(a)

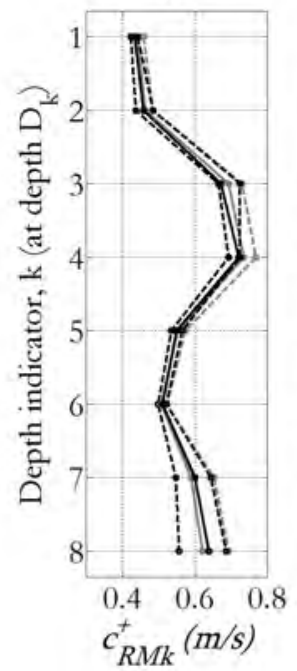

(b)

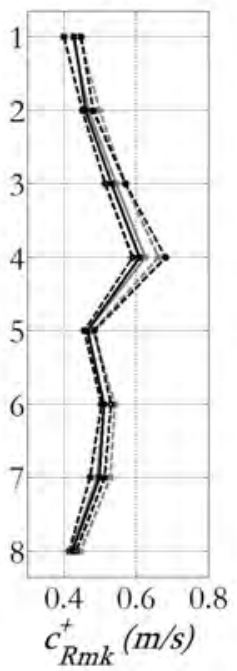

(c)

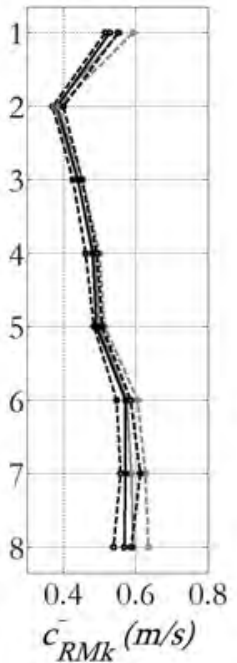

(d)

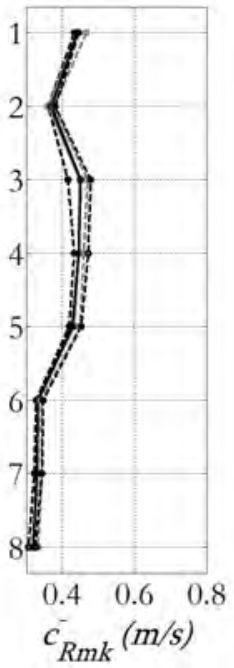

Figure 11: Median marginal 10-year return values for hourly extreme residual currents with depth $(k=$ $1,2, \ldots, 8)$ estimated from conditional extremes simulation (solid black) and theory using fitted marginals (solid grey), for (a) $c_{R M k}^{+}$, (b) $c_{R m k}^{+}$, (c) $c_{R M k}^{-}$and (d) $c_{R m k}^{-}, k=1,2, \ldots, 8$. Also shown are corresponding $25 \%$ iles and $75 \%$ iles (dashed). 
(a)

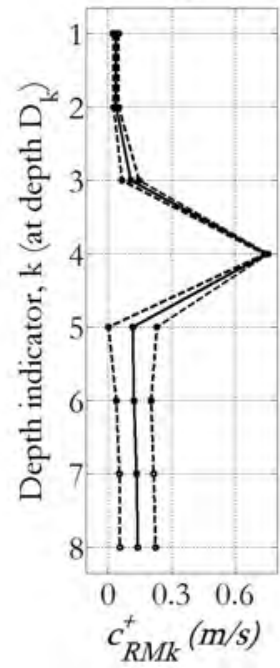

(b)

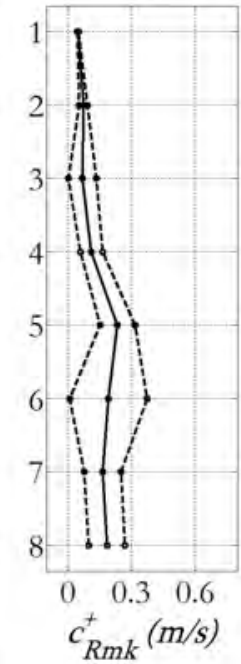

(c)

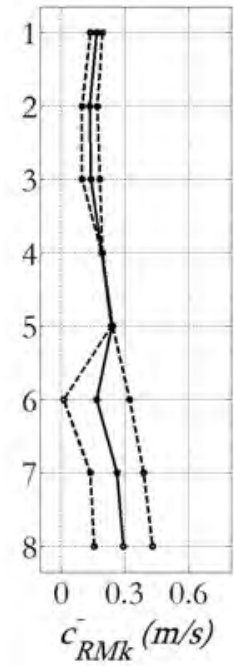

(d)

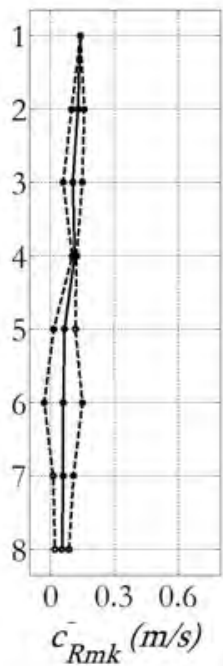

Figure 12: Median (solid) conditional hourly extreme residual current profiles with depth $(k=1,2, \ldots, 8)$ for exceedences of the 10-year return level of $c_{R M 4}^{+}$, estimated from conditional extremes simulation for (a) $c_{R M k}^{+}$, (b) $c_{R m k}^{+}$, (c) $c_{R M k}^{-}$and (d) $c_{R m k}^{-}, k=1,2, \ldots, 8$. Also shown are corresponding $25 \%$ iles and $75 \%$ iles (dashed).

(a)

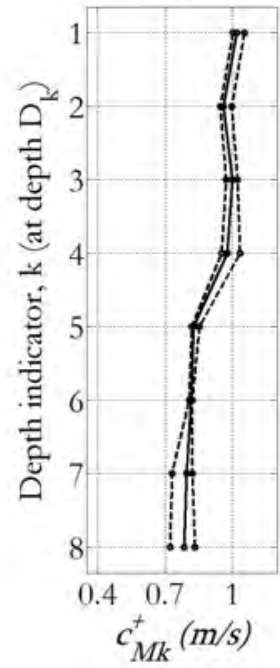

(b)

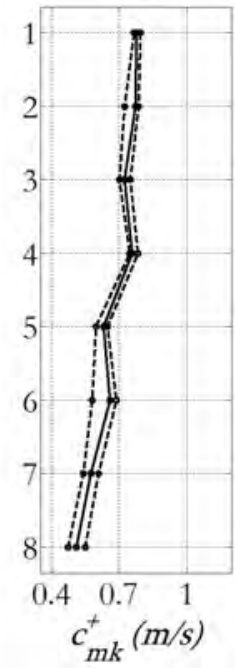

(c)

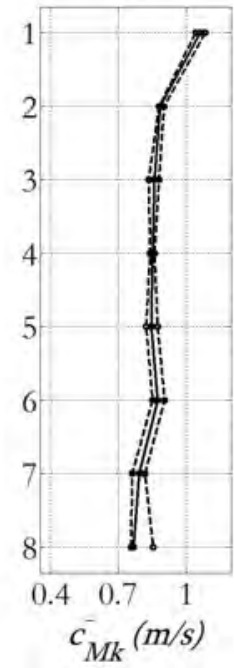

(d)

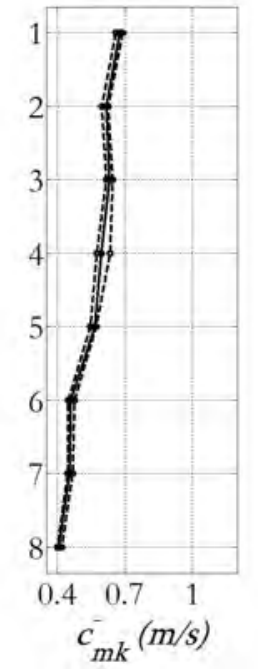

Figure 13: Median marginal 10-year return values for hourly extreme total currents with depth $(k=1,2, \ldots, 8)$ estimated from full conditional extremes simulation (solid black) and theory using fitted marginals (solid grey), for (a) $c_{M k}^{+}$, (b) $c_{m k}^{+}$, (c) $c_{M k}^{-}$and (d) $c_{m k}^{-}, k=1,2, \ldots, 8$. Also shown are corresponding $25 \%$ iles and $75 \%$ iles (dashed). 
(a)

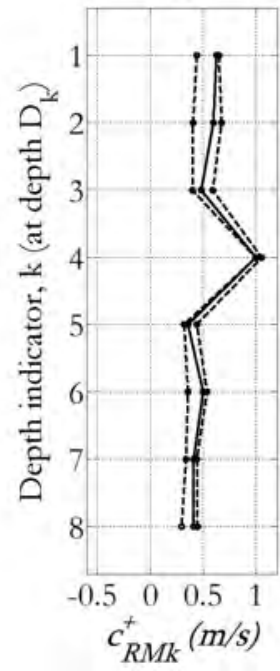

(b)

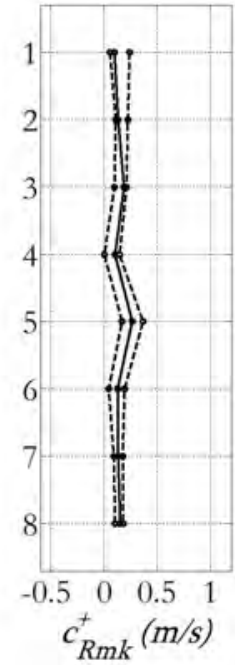

(c)

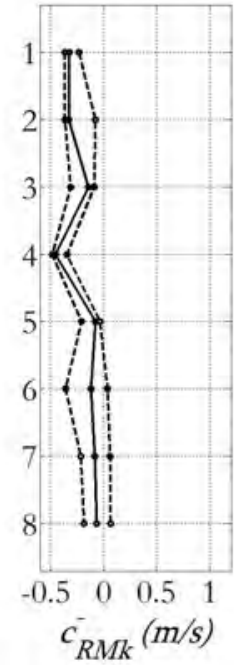

(d)

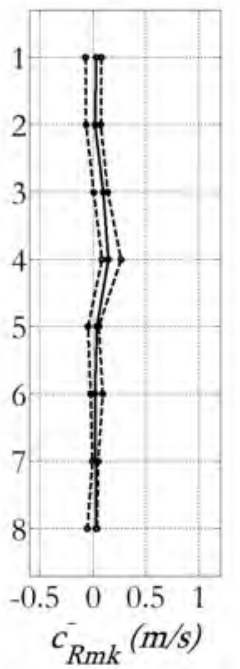

Figure 14: Median (solid) conditional hourly extreme total current profiles with depth $(k=1,2, \ldots, 8)$ for exceedences of the 10-year return level of $c_{M 4}^{+}$, estimated from full conditional extremes simulation for (a) $c_{M k}^{+}$, (b) $c_{m k}^{+}$, (c) $c_{M k}^{-}$and (d) $c_{m k}^{-}, k=1,2, \ldots, 8$. Also shown are corresponding $25 \%$ iles and $75 \%$ iles (dashed). Note that difference y-axis scales are used .

of the 10-year return level of $c_{M 4}^{+}$. We note that conditional values for other major-axis maxima $\left(c_{M k}^{+}, k=\right.$ $1,2, \ldots, 8, k \neq 4)$ are relatively large, with medians of $0.6 \mathrm{~ms}^{-1}$ at $D_{1}$ falling to $0.4 \mathrm{~ms}^{-1}$ at $D_{8}$. In contrast, values of (negative) major-axis minima $\left(c_{M k}^{+}, k=1,2, \ldots, 8\right)$ are themselves negative, indicating that even the minimum of this current component is in the direction of the (positive) major axis at each depth. Conditional minor-axis maxima and (negative) minima are relatively small and similar in values. For comparison with Figure 14(a), Figure 15 shows the conditional profiles of $c_{M k}^{+}$with depth $(k=1,2, \ldots, 8)$ for exceedences of the 10-year return level of $c_{M 1}^{+}, c_{M 4}^{+}$and $c_{M 8}^{+}$. The dependence between components at depths $D_{1}-D_{3}$ (for conditioning on $c_{M 1}^{+}$), and depths $D_{7}$ and $D_{8}$ (for conditioning on $c_{M 8}^{+}$) is apparent.

Figures 16 and 17 present estimates of marginal and conditional monthly maxima of current components, estimated both from conditional simulation and directly from measured data. Note that values from a simulation of hourly extremes cannot be directly compared with values from measurements (sampled at 1minute intervals). Nevertheless, we might expect that hourly extremes would provide bounds for the values from minute measurements, as discussed below. Figure 16 illustrates conditional total current profiles $c_{M k}$ with depth $(k=1,2, \ldots, 8)$ for monthly maxima of $c_{M 1}, c_{M 4}, c_{M 6}$ and $c_{M 8}$. Agreement between (hourly) simulation and (minute) measurements is good in terms of general profiles in each case. Moreover, simulation appears to provides an upper bound for measured profile. This can be rationalised as follows: the measured profile, conditional on a monthly maximum at a particular depth, quantifies the profile observed during that minute of observation. In contrast, the corresponding simulated profile quantifies the profile of the maximum values observed during the hour corresponding to the occurrence of the monthly maximum. Figure 17 gives conditional total current minor-axis profiles with depth for the same conditioning variates as Figure 16 estimated from conditional simulation and measured minute profiles. We can use simulated profile $\left\{c_{m k}^{+}\right\}_{k=1}^{8}$ as an upper bound for the measured profile, and simulated profile $\left\{-c_{m k}^{-}\right\}_{k=1}^{8}$ (not negative sign) as a lower bound.

\section{Discussion and conclusions}

In this paper, we consider an approach to modelling the vertical (vector) profile of large ocean currents, using the conditional extremes model introduced to the statistical literature by Heffernan and Tawn 2004]. The key steps in the analysis are summarised in section 4.1. Observed vector currents at each of 8 depths 
(a)

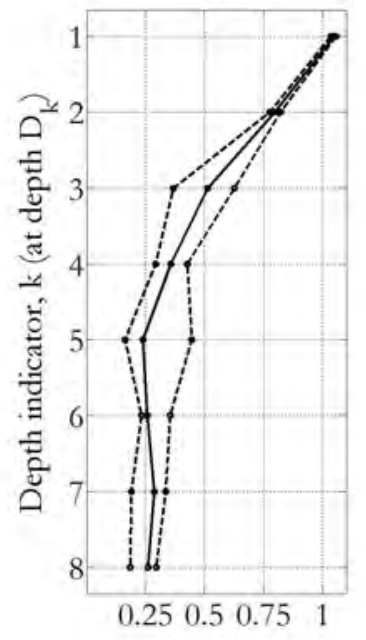

(b)

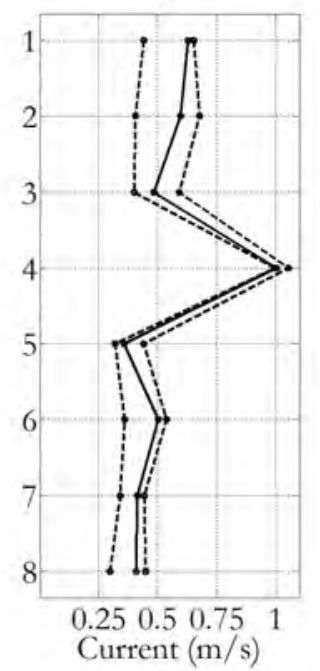

(c)

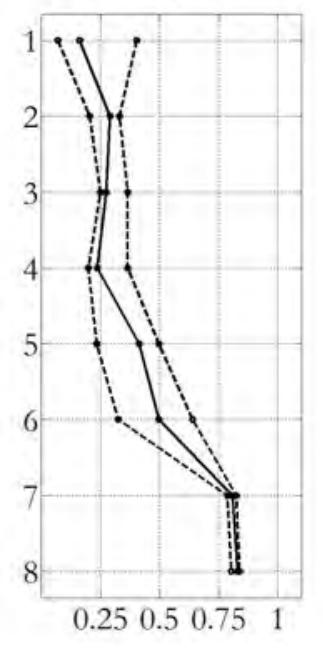

Figure 15: Median (solid) conditional hourly extreme total current profiles along major axis, $c_{M k}^{+}$, with depth $(k=1,2, \ldots, 8)$ for exceedences of 10-year return level of (a) $c_{M 1}^{+}$, (b) $c_{M 4}^{+}$and (c) $c_{M 8}^{+}$, estimated from conditional extremes simulation. Also shown are corresponding $25 \%$ iles and $75 \%$ iles (dashed).

(a)

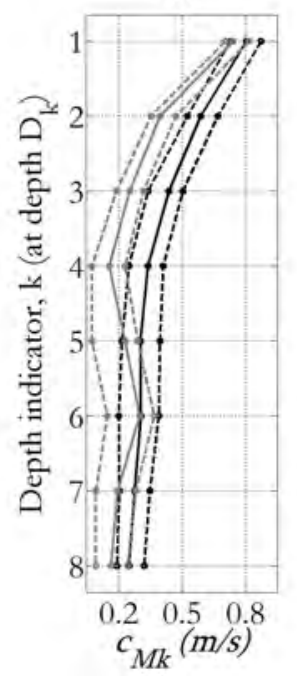

(b)

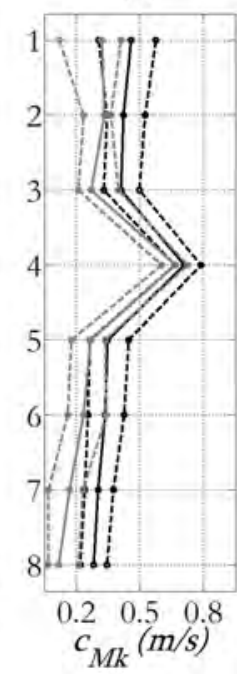

(c)

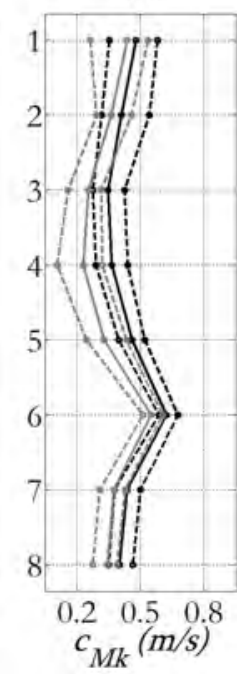

(d)

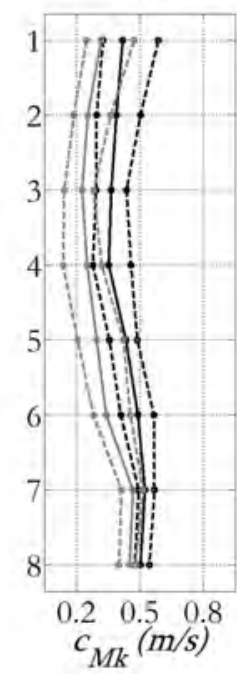

Figure 16: Median (solid) conditional total current profiles along major axis, $c_{M k}$, with depth $(k=1,2, \ldots, 8)$ for monthly maxima of (a) $c_{M 1}$, (b) $c_{M 4}$, (c) $c_{M 6}$ and (d) $c_{M 8}$ estimated from conditional simulation of hourly extremes (black) and measurements of measured profiles (grey). Also shown are corresponding $25 \%$ iles and $75 \%$ iles (dashed). Conditional profiles from simulation provide an upper bound for the instantaneous profile. 
(a)

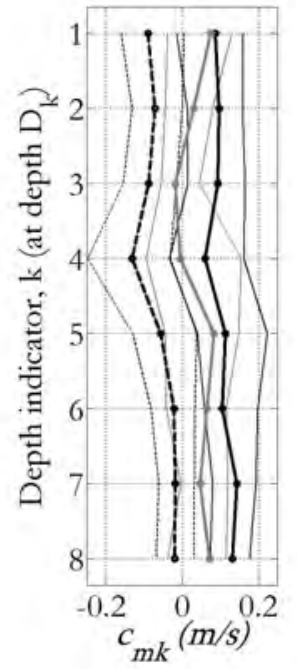

(b)

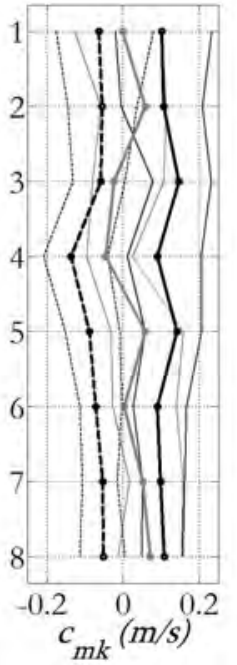

(c)

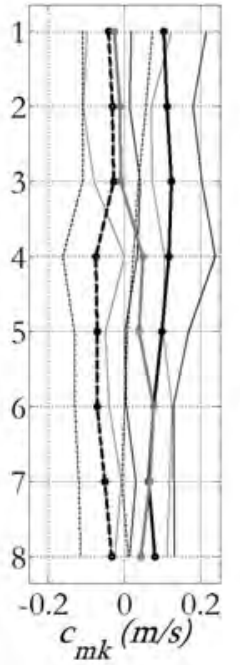

(d)

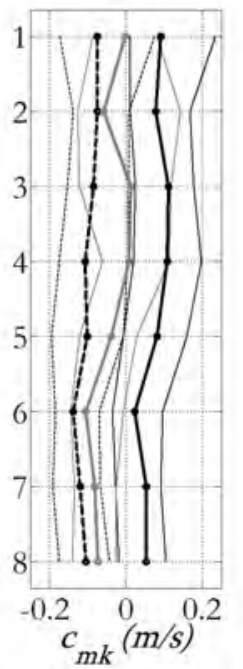

Figure 17: Median conditional total current profiles along minor-axis, $c_{m k}$, with depth $(k=1,2, \ldots, 8)($ thick lines) for monthly maxima of (a) $c_{M 1}$, (b) $c_{M 4}$, (c) $c_{M 6}$ and (d) $c_{M 8}$ estimated from conditional simulation of hourly extremes of $c_{m k}^{+}$(black solid) and $-c_{m k}^{-}$(black dashed), and measurements of minute profiles (grey). Also shown are corresponding 25\%iles and $75 \%$ iles (dashed). Conditional profiles from simulation provide useful bounds for the measured profile.

are expressed as components with respect to major- and minor-axis of current variation at that depth using principal components analysis. Resolved currents are then each decomposed into tidal and non-tidal components. Marginal and dependence structure of extremes of hourly maxima and minima of non-tidal components is then characterised using the conditional extremes model. We simulate under this model to estimate characteristics of extreme current profiles corresponding to arbitrary return periods, and quantify the uncertainty of those estimates. We demonstrate that the model predicts monthly instantaneous extreme conditional profiles well for the sample of Western Australian data considered, and provide marginal and conditional current profiles corresponding to a 10-year return period corresponding to that location.

Modelling the marginal and dependence structure of extremes of vector currents over multiple depths is a complex issue, not readily amenable to statistical analysis. For offshore design, estimation of extreme current profiles has been approached in a number of ways. In shallower water, it has been assumed that current profile through depth follows some nominal profile, approximated by a $1 / 7$ th power law or logarithmic function (e.g. ISO 2005]). In deeper water, current measurements through the water column are generally more readily available, allowing empirical modelling from these observations. One approach is to assume that currents at each depth are independent of the remainder of the profile, and then apply extreme value analysis at each depth. Forristall and Cooper [1997] apply Principal Component Analysis (Empirical Orthogonal Functions) and the First Order Reliability Method (FORM) analyse current profiles in West Africa and the Atlantic margin; this approach has become standard for determining extreme current profiles in deeper water in the oil and gas industry. In practice, the approach has been to use just the first two or three modes to arrive at the extreme conditions, but in many situations this is insufficient (see, e.g. Meling et al. 2002, Jeans and Cooper 2005).

The modelling approach provides a basis for simulating (multivariate) time-series of currents with representative marginal and dependence structures. These can be used to address oceanographic and engineering issues. For example, results of the modelling approach have been summarised here in terms of conditional current profiles. From a structural design perspective, simulated current time-series can be used to estimate current design profiles corresponding to long return periods, or extreme characteristics of structure variables such as structural response to current forcing (see, e.g., Heffernan and Tawn 2004, Ewans and Jonathan 2008). The analysis may be framed in terms of economics if appropriate cost (or loss) functions are available. 


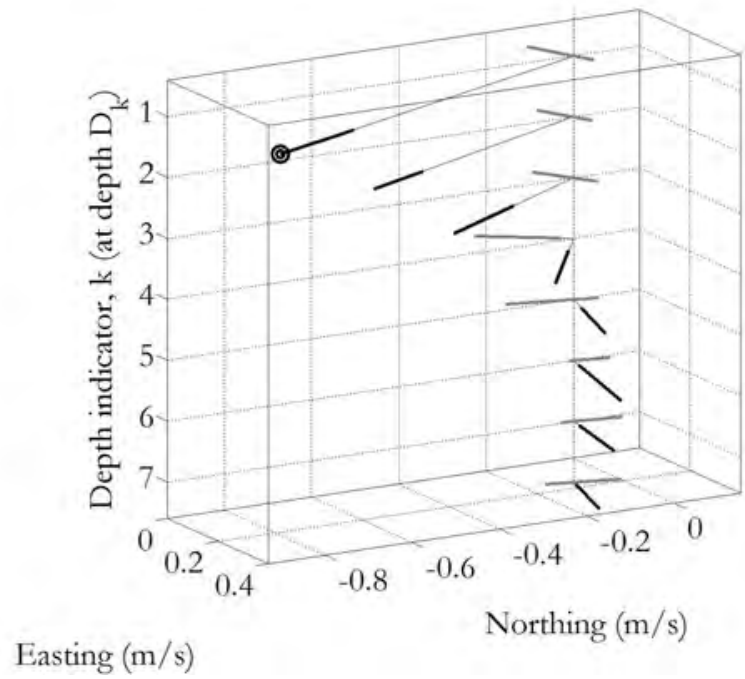

Figure 18: Illustration of median conditional total current components with depth $(k=1,2, \ldots, 8)$ for exceedences of 10-year return level of $c_{M 1}^{+}$from conditional simulation of hourly extremes. The interval $\left(-c_{M k}^{-}, c_{M k}^{+}\right)$is projected onto the positive major-axis (black), and $\left(-c_{m k}^{-}, c_{m k}^{+}\right)$is projected onto the positive minor-axis (grey), at each depth $k$. The figure suggests systematic rotation of currents clockwise with respect to unconditioned currents at depth $D_{4}$, but anti-clockwise rotation at larger depths.

We believe that the approach introduced in the present article represents an interesting pragmatic addition to the modeller's toolbox. In particular, use of localised harmonic analysis circumvents difficulties in accommodating temporal variability of the ocean's flow response to tidal forcing, and the use of hourly maxima of components along the principal and orthogonal axis provides useful engineering parameters whilst preserving temporal dependence. The main advantage of the method is that the extreme value estimation is theoretically underpinned by the conditional extremes model, providing a rational framework for modelling extremes in high-dimensions. In common with any statistical modelling, we assume that the sample of current data for analysis is representative, so that estimates of extreme conditions are also representative, particularly with respect to rare events (e.g. tropical cyclones). As presently implemented, the approach makes a number of further assumptions.

Firstly, we assume that it is appropriate to consider analysis of current components (at each depth) corresponding to major- and minor- axes of current variation at each water depth. Modelling with uncorrelated components at each depth would seem desirable statistically, as would examining component corresponding to major axis of current variation at each depth (in the spirit of principal components analysis). Physical considerations of on-offslope and along-slope currents might also support this. However, other choices of current components might be considered reasonable. Since in general the estimates of extreme currents made using the approach will depend on this choice, it should be made with care, and the sensitivity of estimates with respect to the choice assessed. It may be the case that the orientation of principal axes at a given depth itself changes as a function of current magnitude. Figure18 shows median conditional total current components with depth $(k=1,2, \ldots, 8)$ for exceedences of 10-year return level of $c_{M 1}^{+}$from conditional simulation, constructed by projecting the interval $\left(-c_{M k}^{-}, c_{M k}^{+}\right)$onto the (positive) major axis direction, and $\left(-c_{m k}^{-}, c_{m k}^{+}\right)$onto the (positive) minor-axis, at each depth $k$. The figure suggests that the minor-axis conditional extremes are approximately symmetric about zero at depths $D_{1}-D_{3}$. At depth $D_{4}$ however, there is systematic rotation of current components in a clock-wise direction, with respect to axis directions defined using unconditioned sample at this depth. At depths $D_{5}-D_{8}$, this trend is reversed; rotation is in an anti-clockwise direction. Similar behaviour is observed when conditioning on exceedences of 10-year return level of $c_{M 1}^{+}$(Figure 19). Subsequent inspection of original data confirms that this effect is present. It may therefore be appropriate to select current components with respect to principal axes for vector currents whose magnitude exceeds some threshold. 


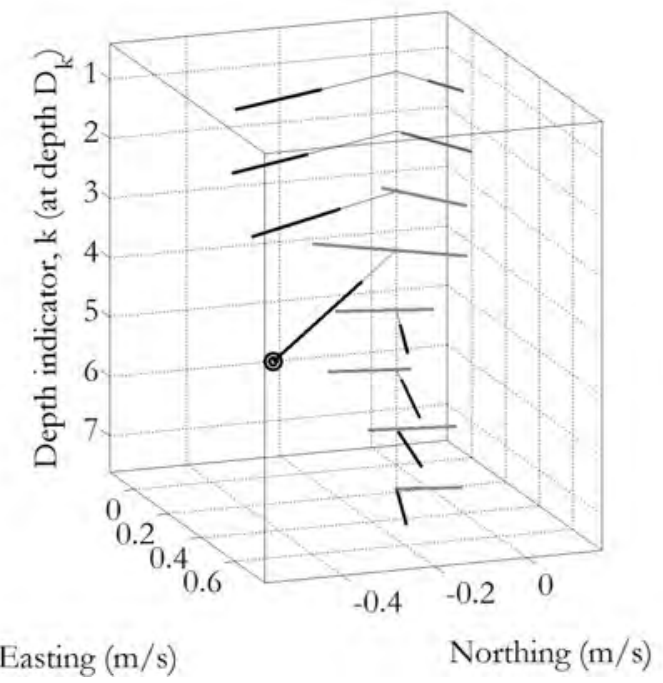

Figure 19: Illustration of median conditional total current components with depth $(k=1,2, \ldots, 8)$ for exceedences of 10-year return level of $c_{M 4}^{+}$from conditional simulation of hourly extremes. The interval $\left(-c_{M k}^{-}, c_{M k}^{+}\right)$is projected onto the positive major-axis (black), and $\left(-c_{m k}^{-}, c_{m k}^{+}\right)$is projected onto the positive minor-axis (grey), at each depth $k$. The figure suggests systematic anti-clockwise rotation of currents with respect to unconditioned currents at larger depths.

Secondly, decomposition of each current component as the sum of tidal (including slowly-varying) and (non-tidal) residual currents using a local harmonic model involves a number of approximations. We assume that independent decomposition of components is appropriate. Once estimated, no further modelling of tidal currents is performed; they are merely (jointly) sampled with replacement during simulation. For estimation of return values of tidal current corresponding to a return period (e.g. 1 month) much shorter than the period of the data sample (e.g. 2.5-years), this might be a reasonable approach. However for return periods longer than the period of the data, longer-term tidal forcing not adequately characterised by the local harmonic model will assume increasing importance. In this situation, it may be useful to add the corresponding incremental tidal current effects to the estimated tidal currents, before sampling during simulation.

Thirdly, the present approach assumes that tidal and residual current components are independent. Hence it is valid to simulate from the (joint) tidal current estimates and the (joint) residual current model independently, before adding the values to obtain an estimate of total current (per component per depth). However, inadequacy and/or uncertainty in the initial decomposition of current components might result in dependence between tidal and residual estimates, invalidating the assumption of independence. Fourthly, since current direction varies considerably, we need to understand marginal and dependence structure of both maxima and minima residual current components. We therefore choose to apply the conditional extremes model to hourly maxima and hourly (negative) minima of residual current components. Yet we seek to estimate effectively instantaneous extreme current profiles. As argued in section 4.3, we expect the model (based on hourly extremes) to provide upper bounds for instantaneous extreme current profiles. Comparison of model estimates with observations of 1-month return periods supports this.

In applying the present approach we should bare in mind that the conditional extremes model is motivated by asymptotic theory. To be strictly valid, it should be applied to modelling the extreme tails of multivariate distributions. Various diagnostic tools discussed in section 3 can be used to seek to justify its adequacy in any application. Threshold selection (from marginal and each pairwise dependence model) is critical. In principle, different threshold choices might be appropriate in each case. For an application involving $p$ variables, this amounts potentially to $p^{2}$ different threshold selections. Automatic threshold selection becomes inevitable; in the present work, we have taken a threshold corresponding to a constant small marginal exceedence probability, which appears reasonable from inspection of a random selection of diagnostics plots. 
Nevertheless, a more satisfactory procedure would be highly desirable.

\section{Acknowledgement}

The authors gratefully acknowledge discussions with colleagues at Shell and Lancaster University, UK, and reviewers' comments.

\section{References}

C. Carollo, L. Astin, and J. Graff. Vertical structure of currents in the vicinity of the Iceland-Scotland ridge. Annales Geophysicae, 23:1963-1975, 2005a.

C. Carollo, L. Astin, and J. Graff. Vertical structure of extreme currents in the Faroe-bank channel. Annales Geophysicae, 23:1977-1986, 2005b.

K. C. Ewans and P. Jonathan. The effect of directionality on Northern North Sea extreme wave design criteria. J. Offshore Mechanics Arctic Engineering, 130:10, 2008.

G. Z. Forristall and C. K. Cooper. Design current profiles using empirical orthogonal function (EOF) and inverse FORM methods. Offshore Technology Conference OTC8267, 1997.

J. Graff. The distribution of current meter measurements due to unusual hydraulic phenomena (paper a3). In International conference on measuring techniques of hydraulic phenomena in offshore, coastal and inland waters, 1986.

A. Hannachi, I.T. Jolliffe, and D.B. Stephenson. Empirical Orthogonal Functions and related techniques in atmospheric science: A review. Int. J. Climatology, 27:1119-1152, 2007.

J. E. Heffernan and J. A. Tawn. A conditional approach for multivariate extreme values. J. R. Statist. Soc. $B, 66: 497,2004$.

ISO. BS EN ISO 19901-1:2005, Petroleum and natural gas industries - specific requirements for offshore structures - Part 1: Metocean design and operating conditions. British Standards Institute, 2005.

G. Jeans and C. Cooper. Characterisation of subsurface jet currents offshore West Africa (volume III, 688-693). In Proc. 27th ISOPE conference, 2005.

I.T. Joliffe. Principal Component Analysis. Springer, 2002.

P. Jonathan, J. Flynn, and K. C. Ewans. Joint modelling of wave spectral parameters for extreme sea states. Ocean Engineering, 37:1070-1080, 2010.

K. M. H. Larsen, B. Hansen, and R. Kristiansen et al. Internal tides in the waters surrounding the Faroe plateau. In Proceedings of ICES 2000 Annual Conference, 2000.

Y. Liu and R. H. Weisberg. Ocean currents and sea surface heights estimated across the West Florida shelf. J. Phys. Oceanogr., 37:1697-1713, 2007.

T.S. Meling, K.J. Eik, and E. Nygrd. An assessment of EOF current scatter diagrams with respect to riser VIV fatigue damage (paper OMAE2002-28062). In Proc. XXth International Conf. on Offshore Mechanics and Arctic Engineering, 2002.

R. Pawlowicz, B. Beardsley, and S. Lentz. Classical tidal harmonic analysis including error estimates in MATLAB using TTIDE. Computers and Geosciences, 28:929-937, 2002.

D. T. Pugh. Tides, surges and mean sea level. A handbook for engineers and scientists. Wiley, 1987.

M. E. Robinson and J. A. Tawn. Statistics for extreme sea currents. Applied Statistics, 46:183-205, 1997. 